\title{
DA PRÁTICA BASEADA EM EVIDÊNCIAS PARA A SAÚDE COLETIVA INFORMADA POR EVIDÊNCIAS: REVISÃO NARRATIVA
}

\section{From evidence-based practice to evidence-informed public health: a narrative review}

\section{De la práctica basada en evidencias para la salud colectiva informada por evidencias: revisión narrativa}

Patrick Alexander Wachholz

Universidade Estadual Paulista - UNESP - Botucatu (SP) - Brasil

Silvana Andre Molina Lima

Universidade Estadual Paulista - UNESP - Botucatu (SP) - Brasil

Paulo Jose Fortes Villas Boas

Universidade Estadual Paulista - UNESP - Botucatu (SP) - Brasil

\section{RESUMO}

Objetivo: Revisar na literatura a descrição dos conceitos e o emprego das evidências científicas no âmbito da Saúde Coletiva (SC). Métodos: Realizou-se uma revisão narrativa utilizando e combinando as palavras-chave "Saúde Pública" OR "Saúde Coletiva"; "Medicina baseada em evidências" e "Política informada por evidências" por meio da consulta as bases de dados Lilacs, SciELO e MedLine. As buscas foram limitadas ao período entre janeiro de 1990 a dezembro de 2016, no idioma português e/ou inglês. A seleção dos estudos foi realizada por dois autores, de modo independente, por meio da leitura do título, resumo e texto completo. Para o processo de síntese, as temáticas encontradas foram agrupadas em três grandes eixos norteadores: as evidências e a prática em saúde, as evidências em SC, e os avanços e desafios da Saúde Coletiva Informada pelas Evidências (SCIE). Resultados: Os achados desta revisão apontam que as tomadas de decisão na SC são mais complexas do que as decisões clínicas individuais, pois envolvem avaliações de impactos orçamentários e políticos, reforçando a relevância da adoção de práticas informadas pelas evidências neste campo. Conclusão: Os meios de apropriação e emprego das evidências no campo da SC são complexos, pois envolvem pressupostos de plausibilidade e adequação nem sempre observados em intervenções individuais. Ainda assim, o campo beneficia-se da aproximação observada na SCIE e, em particular, na tomada de decisões em saúde.

Descritores: Saúde Pública; Medicina Baseada em Evidências; Tomada de Decisões.

\section{ABSTRACT}

Objective: To review the literature about the description of concepts and the use of scientific evidence in Public Health (PH). Methods: A narrative review was carried out using and combining the keywords "Public Health" OR "Collective Health"; "Evidence-based medicine" and "Evidence-informed policy" to consult the Lilacs, SciELO and MedLine databases. The searches were limited to articles published between January 1990 and December 2016 in Portuguese and/or English. The studies were selected by two independent authors who read the title, abstract and full text. For the synthesis process, the themes found were grouped into three main guiding axes: health evidence and practice, PH evidence, and the advances and challenges of Evidence-Informed Public Health (EIPH). Results: The findings of this review point out that decision making in PH is more complex than individual clinical decisions as it involves assessment of budgetary and political impacts, thus reinforcing the relevance of adopting evidence-informed practice in this field. Conclusion: The means of appropriation and use of evidence in the PH field are complex because they involve assumptions of plausibility and adequacy that are not always observed in individual interventions. Yet, the field benefits from the approach observed in EIPH and, particularly, in health decision making.

Descriptors: Public Health; Evidence-based Medicine; Decision Making. 


\section{RESUMEN}

Objetivo: Revisar en la literatura la descripción de los conceptos y el empleo de las evidencias científicas en el ámbito de la Salud Colectiva (SC). Métodos: Se realizó una revisión narrativa utilizando y mesclando las palabras-clave "Salud Pública" OR "Salud Colectiva"; "Medicina basada en la evidencia" AND "Política informada por evidencias" a través de la consulta en las bases de datos LILACS, SCiELO y MEDLINE. Las búsquedas se han limitado al periodo entre enero de 1990 y diciembre de 2016 en los idiomas portugués y/o inglés. La selección de los estudios se dio por dos autores de modo independiente a través de la lectura del título, resumen y texto completo. Para el proceso de sintesis se han unido las temáticas encontradas en tres grandes ejes norteadores: las evidencias y la práctica en salud, las evidencias en SC y los avances y desafios de la Salud Colectiva Informada por las Evidencias (SCIE). Resultados: Los hallazgos de esta revisión apuntan que las tomas de decisiones de la SC son más complejas que las decisiones clínicas individuales pues implican evaluaciones de impactos políticos y de presupuesto con refuerzo para la importancia de prácticas informadas por las evidencias en este campo. Conclusión: Los medios de apropiación y empleo de las evidencias para el campo de la SC son complejos pues implican presupuestos de plausibilidad y adecuación no observados en intervenciones individuales. Aun así, el campo se beneficia de la aproximación observada en la SCIE y, en particular, de las tomas de decisiones en salud.

Descriptores: Salud Pública; Medicina Basada en la Evidencia; Toma de Decisiones.

\section{INTRODUÇÃO}

Um dos desafios que se impõe para a formulação e implementação de políticas públicas de saúde é a promoção da utilização de evidências científicas, em países como o Brasil, onde estas sofrem fortes influências de fatores socioeconômicos e da limitação orçamentária, que são desafios ainda maiores ${ }^{(1,2)}$.

A adoção de evidências científicas (EC) na prática da Saúde Coletiva (SC) tem como objetivo garantir a otimização de recursos e a maior efetividade nas ações de promoção, prevenção e atenção à saúde ${ }^{(1)}$. A despeito do uso de evidências na SC ser possível e desejável, a transposição do conceito "baseados em evidências" para o campo não é direta. As complexas ações envolvidas na determinação social das doenças e no processo de trabalho em saúde, típicos da SC, diferem substancialmente da simplificação usualmente envolvida nas práticas individuais de saúde, exemplificadas pelo atendimento individual em consultório médico principalmente.

Um estudo ${ }^{(2)}$ é categórico ao afirmar que o uso das EC pelos gestores de saúde ainda é elementar, principalmente quando se analisa o emprego destas na "tomada de decisão"(2).

Com o intuito de discutir as principais dificuldades inerentes a esse processo e apresentar iniciativas já existentes que possam permitir uma melhor aproximação, o objetivo deste estudo foi revisar na literatura a descrição dos conceitos e o emprego das evidências científicas no âmbito da Saúde Coletiva.

\section{MÉTODOS}

Para a realização desta revisão narrativa foi conduzida uma busca na literatura, utilizando as palavras-chave (e seus principais sinônimos) dos Descritores em Ciências da Saúde (DeCS): "Saúde Pública" OR "Saúde Coletiva", "Medicina baseada em evidências" e "Política informada por evidências". Foram consultadas as bases de dados Lilacs, SciELO e MedLine (via PubMed). As buscas, realizadas por dois autores de modo independente, foram limitadas ao período entre janeiro de 1990 a dezembro de 2016, e restritas a artigos publicados em português e/ou inglês.

Os critérios de seleção aplicados incluíram: estudos qualitativos, quantitativos, de revisão ou editoriais; cujo tema central tenha sido a análise da relação entre o uso das evidências científicas e seu emprego e/ou impacto nas políticas públicas de saúde. Os títulos e resumos de 29.835 citações foram revisados (excluindo-se as duplicadas), e o texto completo de 34 manuscritos foram lidos e considerados para redação desta revisão.

Para o processo de síntese, dois autores agruparam os temas mais prevalentes nos manuscritos incluídos, identificando três grandes eixos norteadores: as evidências e a prática em saúde; as evidências em Saúde Coletiva e; os avanços e desafios da Saúde Coletiva Informada pelas Evidências. A partir desses tópicos, discutir-se-ão os achados desta revisão.

\section{RESULTADOS}

\section{As evidências e a prática em saúde}

O conceito de "evidência" surge por meio de René Descartes, na primeira metade do século XVII, a partir da obra "Discours de la méthode". Nela, o filósofo transmite sua preocupação por encontrar um método para conhecer a verdade que, ao ser utilizado, permitisse aceitar como verdadeiro apenas aquilo que fosse evidente ${ }^{(3)}$. 
Contemporaneamente, o termo "evidências científicas" tem sido empregado como o conjunto de informações utilizadas para confirmar ou negar uma teoria ou hipótese científica. Desse modo, evidências são produzidas quando estudos e pesquisas são conduzidos com a intenção de esclarecer a relação, efeito ou causalidade entre duas ou mais variáveis, condições ou intervenções.

As primeiras referências ao termo "Medicina Baseada em Evidências" (MBE) foram publicadas por Guyatt e Sackett(t,5), na época eminentes epidemiologistas clínicos na Universidade de McMaster (Canadá). Posteriormente, seu grupo de discípulos estruturaram e fomentaram a disseminação do conceito, atraindo protagonistas, principalmente na América do Norte ${ }^{(\bullet)}$. A MBE surge, então, como um movimento científico que se traduz pela recomendação de que prática da medicina deva acontecer em um contexto em que a experiência profissional integre-se com a capacidade de analisar criticamente a informação científica disponível e aplicá-la de forma racional, em parceria com o(s) sujeito(s) envolvido(s), de forma a melhorar a qualidade da assistência ${ }^{(7)}$.

Sem pretensões niilistas, a MBE caracterizou-se por recomendar o uso judicioso e explícito da melhor EC disponível nas tomadas de decisão, sempre com foco no cuidado individual com a saúde ${ }^{(5,6,8)}$. Ainda que distâncias importantes viessem a ser reconhecidas entre as evidências geradas pelas análises epidemiológicas e a prática em saúde, a MBE forneceu importantes subsídios, os quais permitiram avanços na validação das informações, na análise da eficácia e na efetividade das intervenções em saúde, bem como da prática médica ${ }^{(9)}$.

Gradualmente, a MBE foi sendo incorporada, apropriada e adaptada a diferentes contextos, cenários e movimentos. O seu construto evoluiu, paulatinamente, para conceito mais amplo de "Prática Baseada em Evidências"(10) (PBE). A evidência, per se, não era capaz de direcionar ou modificar as decisões em saúde, ou seja, a PBE partia do pressuposto de que os tomadores de decisão deveriam levar em conta as necessidades e valores do sujeito (e também das populações), bem como a disponibilidade de recursos para sua implementação ${ }^{(11)}$.

Devido ao incomensurável aumento no volume de informações e evidências potencialmente disponíveis para uso na prática profissional em nível individual, bem como para a estruturação das políticas públicas e/ou decisões operacionais em saúde, a PBE destacou-se como uma alternativa pertinente e atraente ${ }^{(6,11)}$. Ao disponibilizar estratégias para a seleção das melhores evidências disponíveis e recomendar o uso eficaz dessas informações, esperava-se fornecer condições para que os gestores públicos pudessem adotar "melhores práticas" nas tomadas de decisão(12,13).

Nesse contexto, é fundamental perceber que "criar evidências" e "criar ações em saúde" são processos absolutamente distintos, sendo as pesquisas científicas (e todas as formas de produzir evidências) consideradas atos de produção do conhecimento. Por outro lado, a criação de ações em saúde (por exemplo, tomada de decisão), que pode utilizar (ou não) as evidências produzidas e disponíveis, constitui-se fundamentalmente em ato político ou resultante das relações sociais de poder ${ }^{(14)}$.

A apropriação e o emprego das EC passaram a ser uma conduta recomendada e preconizada pela Organização Mundial de Saúde ${ }^{(15)}$ (OMS), com vistas a melhorar o desempenho dos sistemas de saúde entre seus membros. Do mesmo modo, a Organização Pan-Americana de Saúde (OPAS) incluiu a aplicação de EC entre as linhas de ação recomendadas para o fortalecimento da capacidade institucional na agenda de saúde para as Américas (2008-2017) ${ }^{(16)}$.

A transferência do conceito "baseado em evidência" da prática clínica para o campo de conhecimentos e práticas da SC, porém, não tem sido tão direta ${ }^{(13)}$. Nesse contexto, SC significa "um esforço organizado da sociedade, principalmente suas instituições públicas, para melhorar, promover, proteger e restaurar a saúde da população mediante ações coletivas"(17).

As evidências parecem condições necessárias, mas não suficientes, para responder às perguntas e necessidades da coletividade ${ }^{(14,18)}$. Além de saber se uma intervenção funcionará (eficácia), mostrou-se ímpar, nesse campo, indagar: se ela funcionaria no "mundo real" (efetividade); a que custo (eficiência); qual seria a distribuição dos riscos e benefícios; se sua implementação seria aceitável e adequada (sem riscos); se a capacidade do sistema de incorporar essa intervenção permitiria sua disponibilização a todos (equidade) ${ }^{(12)}$.

Enquanto a MBE e a PBE direcionam seu foco principal para a prática individual, a SC toma como objeto as necessidades sociais de saúde voltadas à coletividade, "concebendo as ações de atenção à saúde como práticas técnicas e sociais" (19). Particularmente em domínios complexos, como a promoção da saúde, aspectos fundamentais (como a "aceitabilidade”, a adesão e a importância outorgada por uma população à determinada intervenção) mostram-se tão ou mais importantes que a qualidade metodológica de uma evidência, sua consistência e corroboração $0^{(3,20)}$.

\section{As evidências no contexto da Saúde Coletiva}

No campo e prática da SC, o construto "Saúde Coletiva baseada em evidências" (SCBE) passou a ser incorporado como um dos instrumentos empregados no ciclo da produção de evidências e respostas necessárias à tomada de decisões ${ }^{(14)}$.

A SCBE fundamenta-se na integração e uso consciente e crítico da melhor evidência corrente disponível para a tomada de decisão sobre a atenção à comunidade e às populações, nos campos da proteção, prevenção de doenças e promoção da saúde ${ }^{(13,14,21)}$.

De fato, para que melhores decisões sobre intervenções em saúde possam ser adotadas, é fundamental que exista um processo transparente e reprodutível, que considere, por exemplo, se uma intervenção é suficientemente incapaz de causar prejuízo mensurável. Geralmente, essas informações são provenientes de evidências geradas por estudos observacionais e, quando possível, avaliadas em nível experimental (por meio de ensaios clínicos randomizados - ECR), para que depois possam ser estimadas análises de custo-efetividade ${ }^{(22)}$. 
Inseridas na complexidade contextual da prática em SC, no entanto, as evidências devem ser consideradas não como uma resposta definitiva para problemas específicos, mas como um elemento adicional a ser incorporado na argumentação e no debate que norteia a tomada de decisões, transcendendo o campo epidemiológico e interagindo com as dimensões social, cultural e político-econômica $^{(14,21)}$.

Conforme assinalado em um estudo(23), considerando que as intervenções em SC estão sujeitas "a um grau de complexidade superior ao das pesquisas clínicas usuais", é importante perceber algumas diferenças fundamentais entre a PBE e a SCBE: a) a unidade de análise geralmente é populacional, e não individual; b) intervenções multicomponentes complexas são, frequentemente, mais difíceis de serem descritas e caracterizadas do que intervenções simples (como o consumo de um medicamento específico em um ambiente controlado de pesquisa); c) e nem sempre os delineamentos aleatorizados e controlados são possíveis em Saúde Coletiva $^{(23)}$.

As decisões em SC tendem a ser mais complexas do que as decisões clínicas, pois envolvem a avaliação do provável impacto das políticas e outras intervenções que são, , em geral, de longo prazo ${ }^{(24)}$. A tomada de decisão em SC não é um evento único, mas um processo difuso, com várias etapas espalhadas ao longo do tempo, sem uma relação clara e previsível entre elas ${ }^{(25)}$.

Apesar da SCBE ser tanto possível quanto desejável, o percurso necessário para que uma intervenção testada sobre condições controladas seja reprodutível sob condições rotineiras é muito mais complexo ${ }^{(20)}$. Pressupostos de probabilidade (baseados estritamente nos resultados de ECR) devem ser complementados por confirmações de plausibilidade (derivadas de delineamentos observacionais com grupos de comparação) e de adequação (efeitos ou tendências no comportamento de indicadores sugerindo a efetividade da intervenção) $)^{(18,20)}$.

Nenhum método de pesquisa é capaz de, individualmente, oferecer respostas suficientes para a imediata aplicação de uma evidência no campo da coletividade ${ }^{(9,10,22,26)}$. Diferentemente da aplicação individual da informação advinda de ECR, a SC deve ser capaz de responder à demanda de populações extremamente heterogêneas, incluindo complexidade e variabilidade que, usualmente, não são generalizáveis a partir do resultado de estudos com foco apenas na eficácia de uma intervenção ${ }^{(9)}$.

Na avaliação das intervenções em SC, os ECR, geralmente, não são suficientes como força motriz para a tomada de decisão. Usualmente, a triangulação de diferentes metodologias de pesquisa se condensa com as EC produzidas por estudos epidemiológicos de elevada validade interna, de modo a embasar as decisões sobre intervenções em saúde ${ }^{(20,26)}$.

Recentemente, a OMS passou a recomendar a incorporação de um sistema padronizado de graduação, análise, desenvolvimento e avaliação das evidências (Grading of Recommendatons Assessment, Development and Evaluaton - Sistema GRADE) por ocasião do estabelecimento de recomendações em saúde pública, bem como no processo de tomadas de decisão em saúde ${ }^{(27)}$. As orientações do Sistema GRADE, por exemplo, incluem e destacam a importância dos estudos observacionais e das pesquisas qualitativas na produção de conhecimento e evidências ${ }^{(28)}$.

Dentre as estratégias sugeridas para o estímulo ao uso de evidências nas tomadas de decisão, destacam-se as revisões sistemáticas (RS), que são estudos secundários que utilizam métodos estruturados e rigorosos para examinar, comparar e sintetizar as evidências empíricas relevantes a uma questão de pesquisa específica ${ }^{(29-31)}$. Tem por objetivo localizar, avaliar criticamente e interpretar todos os estudos disponíveis para uma questão de pesquisa, área do conhecimento ou fenômeno de interesse ${ }^{(32)}$.

Ao analisar toda a evidência disponível através de um processo transparente, explícito, reprodutível, rigoroso e abrangente, as RS reduzem os riscos de viés e erros randômicos ${ }^{(22,29,30)}$. As RS permitem a identificação da natureza e da qualidade das evidências disponibilizadas por estudos primários, a consistência do efeito estudado e a evidência de causalidade, especialmente quando os resultados de múltiplos estudos são controversos ou conflitantes ${ }^{(29)}$.

A despeito das RS contribuírem sobremaneira com a construção do conhecimento na SCBE, reduzindo o erro e melhorando a acurácia na tomada de decisões em saúde ${ }^{(10,22,26)}$, autores sugerem que delineamentos alternativos e inovadores devam ser empregados e testados ${ }^{(10,18,20)}$. Dentre estes, destacam-se os delineamentos randomizados e os não-randomizados por cluster e as pesquisas qualitativas ${ }^{(18)}$, assim como as revisões relacionadas a fatores de risco e prognósticos, que incluem estudos observacionais comparativos nos quais o pesquisador coleta dados, observando e comparando os eventos enquanto eles acontecem ${ }^{(32,33)}$.

\section{A Saúde Coletiva Informada pelas Evidências: avanços e obstáculos}

A SC propõe intervenções articuladas de promoção, proteção, recuperação e reabilitação da saúde com base em uma abordagem multidisciplinar ${ }^{(19)}$, com foco nas populações e comunidades (ao invés de em indivíduos), mediante intervenções multicomponentes (ao invés de intervenções únicas); analisando não só os desfechos, mas igualmente o processo causal, as teorias e as crenças das comunidades ${ }^{(18)}$. Nesse contexto, é ímpar que sua complexidade encontre reflexo e respaldo nos meios particulares de apropriação e emprego das evidências científicas.

Tendo em vista que as evidências científicas não determinam as ações e necessidades sociais de saúde, o emprego do conceito de "Saúde Coletiva Informada pelas Evidências" (SCIE) mostra-se mais apropriado do que o termo SCBE. Ainda que as evidências constituam apenas um dos elementos no processo decisório, o uso sistemático das evidências oriundas dos resultados de pesquisas nesse processo é escasso e incipiente, e representa um desafio para as políticas de saúde, principalmente em países em desenvolvimento ${ }^{(2)}$. 
Com o objetivo de promover mecanismos que facilitem o uso regular dos resultados de pesquisas nas tomadas de decisão, respeitando as necessidades locais e o uso racional dos recursos disponíveis, a OMS e a OPAS conceberam uma iniciativa inovadora denominada The Evidence informed policy network (EVIPNet). A rede tem estado presente em 12 países latinoamericanos desde o ano de 2012 (incluindo o Brasil), agindo como facilitadora na revisão de políticas públicas, bem como na condução de diálogos deliberativos, como na prevenção e controle da dengue no espaço urbano e no papel da atenção primária na abordagem compreensiva do manejo de doenças crônicas não-transmissíveis ${ }^{(34)}$.

Independente do contexto de desenvolvimento de uma sociedade, o contínuo desenvolvimento de inovações tecnológicas pode representar um problema para a gestão em saúde, visto que a rápida difusão da informação técnico-científica (sob a influência da indústria, principalmente) cria fortes demandas de incorporação dessas inovações, de forma que os custos dos sistemas de saúde (públicos e/ou privados) tendem a aumentar significativamente. Infelizmente, uma inovação tecnológica não necessariamente representa economia de recursos ou incremento perceptível nos desfechos de interesse para a coletividade.

Nesse sentido, já a partir da década de sessenta, a comunidade internacional passou a identificar a necessidade de constituir processos válidos para auxiliar a tomada de decisão por parte dos gestores em saúde, assim como dos profissionais de saúde, das chefias de serviços, das organizações de pacientes, do sistema judiciário e dos ministros de saúde. Surge, então, o conceito de avaliação de tecnologias em saúde (ATS), que trata de "um processo que avalia e regula o uso das tecnologias em saúde, oferecendo subsídios técnicos baseados na melhor evidência científica"(35).

A ATS emprega como ferramenta fundamental a avaliação crítica da validade das pesquisas clínicas realizadas com a tecnologia de interesse (medicamentos, procedimentos, equipamentos e sistemas organizacionais e de suporte), e é realizada mediante a revisão sistemática da literatura, seguida da análise do custo-efetividade da potencial incorporação da tecnologia ${ }^{(35)}$.

No âmbito do Sistema Único de Saúde (SUS), a Lei nº 12.401/2011, que dispõe sobre a assistência terapêutica e a incorporação de tecnologias em saúde, atribuiu à Comissão Nacional de Incorporação de Tecnologias (CONITEC) a função de incorporação, exclusão ou alteração dessas tecnologias no SUS.

A criação da Rede Brasileira de Avaliação de Tecnologias em Saúde (REBRATS), institucionalizada pela Portaria n ${ }^{\circ} 2.915$, de 12 de dezembro de 2011, foi outro marco importante no estabelecimento de diretrizes públicas voltadas à geração e à síntese de evidências científicas no campo de ATS. A Rebrats é constituída por uma rede de centros colaboradores e instituições de ensino e pesquisa, e encampou o trabalho de padronização e elaboração de diretrizes metodológicas para a ATS no país.

Realizar uma adequada avaliação crítica da validade, integridade e aplicabilidade das evidências pertinentes a intervenções em SC pode ser desafiador, pois nem sempre os processos de randomização, sigilo da alocação e cegamento são factíveis nos delineamentos, e os potenciais vieses de informação e contaminação dos grupos controle/intervenção, aliados ao elevado risco de abandono, podem influenciar a avaliação da efetividade e usualmente comprometem a qualidade final desses estudos ${ }^{(18)}$. Ainda assim, a SCIE é fonte importante de informação para otimizar o uso dos recursos em saúde e assegurar que a tomada de decisão esteja baseada em práticas efetivas e corroboradas.

\section{CONCLUSÃO}

A despeito dos conceitos "baseados em evidência" terem evolú́do desde seus primeiros registros, de um foco no cuidado individual com a saúde para uma conceituação mais ampla de Prática Baseada em Evidências, sua transposição para o campo de práticas da Saúde Coletiva não tem sido direta. Em Saúde Coletiva, os meios de apropriação e emprego das evidências mostram-se muito mais complexos do que o envolvimento nas decisões clínicas, pois envolvem pressupostos de plausibilidade e adequação que nem sempre são observados em intervenções individuais, bem como avaliações de impactos orçamentários e políticos.

Ainda assim, o campo beneficia-se da aproximação observada na Saúde Coletiva Informada pelas Evidências e, em particular, na tomada de decisões em saúde. Iniciativas globais e regionais têm demonstrado a importância desse olhar para otimizar práticas e tornar as ações em saúde mais efetivas.

\section{REFERÊNCIAS}

1. Ministério da Saúde (BR). Síntese de evidências para políticas de saúde: estimulando o uso de evidências científicas na tomada de decisão. Brasilia: Ministério da Saúde; 2015.

2. Dias RISC, Barreto JOM, Vanni T, Candido AMSC, Moraes LH, Gomes MAR. Estratégias para estimular o uso de evidências científicas na tomada de decisão. Cad Saúde Colet (Rio J). 2015;23(3):316-22.

3. Juarez Garcia MS. Nivel de evidencia en salud pública. Rev Soc Peru Med Interna. 2006;19(2):55-7.

4. Evidence-Based Medicine Working Group. Evidence-based medicine: a new approach to teaching the practice of medicine. JAMA. 1992;268(17):2420-5. 
5. Sackett DL, Rosenberg WM. On the need for evidence-based medicine. J Public Health Med. 1995;17(3):330-4.

6. Jenicek M. Epidemiology, evidenced-based medicine, and evidence-based public health. J Epidemiol. 1997;7(4):187-97.

7. Lopes AA. Medicina baseada em evidências: a arte de aplicar o conhecimento científico na prática clínica. Rev Assoc Méd Bras. 2000;46(3):285-8.

8. Glasziou P, Longbottom H. Evidence-based public health practice. Aust N Z J Public Health. 1999;23(4):436-40.

9. Shelton JD. Evidence-based public health: not only whether it works, but how it can be made to work practicably at scale. Glob Health Sci Pract. 2014;2(3):253-8.

10. Ammerman A, Smith TW, Calancie L. Practice-based evidence in public health: improving reach, relevance, and results. Annu Rev Public Health. 2014;35:47-63.

11. Gray JA. Evidence-based public health--what level of competence is required? J Public Health Med. 1997;19(1):65-8.

12. Mowat D. Evidence-based decision-making in public health. Ethos Gub. 2007;4:231-48.

13. Cediel-Becerra NM, Krause G. Evidence-based public health decision-making tools which can also be used for prioritising disease. Rev Salud Pública. 2013;15(5):694-706.

14. Ministério da Saúde (BR), Universidade Federal de Goiás. ASIS - Análise da situação de saúde [Internet]. Brasília: Ministério da Saúde; 2015. v. 1 [acesso em 2015 Jan 12]. Disponível em: http://bvsms.saude.gov.br/bvs/publicacoes/asis_analise_ situacao_saude_volume_1.pdf

15. Tandon A, Murray CJ, Lauer JA, Evans DB. Measuring overall health system performance for 191 countries [Internet]. (Global Programme on Evidence Discussion Paper Series, 30) [acesso em 2016 Out 21]. Disponível em: http://www.who. int/healthinfo/paper30.pdf

16. Pan American Health Organization. Health Agenda for the Americas 2008-2017 [Internet]. Panama: 2007 [acesso em 2016 Out 21]. Disponível em: http://new.paho.org/hq/dmdocuments/2009/Health_Agenda_for_the_Americas_2008-2017.pdf

17. Pan American Health Organization. Public health in the Americas: conceptual renewal, performance assessment and bases for action. Washington: WHO Regional Office for the Americas; 2003.

18. Buendía-Rodríguez JA, Sánchez-Villamil JP. Using systematic reviews for evidence-based health promotion: basic methodology issues. Rev Salud Pública. 2006;8(Supl 2):94-105.

19. Souza LEPF. Saúde pública ou saúde coletiva? Espaç Saúde. 2014;15(4):7-21.

20. Victora CG, Habicht J-P, Bryce J. Evidence-based public health: moving beyond randomized trials. Am J Public Health. 2004;94(3):400-5.

21. Duarte EC. A informação, a análise e a ação em saúde. Epidemiol Serv Saúde. 2003;12(2):61-2.

22. Threlfall AG, Meah S, Fischer AJ, Cookson R, Rutter H, Kelly MP. The appraisal of public health interventions: the use of theory. J Public Health (Oxf). 2015;37(1):166-71.

23. Vidal EIO. O que esperamos das revisões sistemáticas no futuro. Cad Saúde Pública [Internet]. 2016 [acesso em 2017 Jul 14];32(9):eED010916. Disponível em: http://www.scielo.br/scielo.php?script=sci_arttext\&pid=S0102$311 X 2016000900101 \& \operatorname{lng}=\mathrm{en}$

24. Mowat DL, Hockin J. Building capacity in evidence-based public health practice. Can J Public Health. 2002;93(1):19-20.

25. Lonas J. Connecting research and policy. Can J Policy Res. 2002;1:140-4.

26. Hatt LE, Chatterji M, Miles L, Comfort AB, Bellows BW, Okello FO. A false dichotomy: RCTs and their contributions to evidence-based public health. Glob Health Sci Pract. 2015;3(1):138-40.

27. World Health Organization. WHO Handbook for guideline development [Internet].. Geneva: WHO; 2012 [acesso em 2016 Ago 4]. Disponível em: http://apps.who.int/iris/bitstream/10665/75146/1/9789241548441_eng.pdf

28. Balshem H, Helfand M, Schünemann HJ, Oxman AD, Kunz R, Brozek J, et al. GRADE guidelines: 3. Rating the quality of evidence. J Clin Epidemiol. 2011;64(4):401-6.

29. Brannon PM, Taylor CL, Coates PM. Use and applications of systematic reviews in public health nutrition. Annu Rev Nutr. 2014;34:401-19. 
30. Cook DJ, Mulrow CD, Haynes RB. Systematic reviews: synthesis of best evidence for clinical decisions. Ann Intern Med. $1997 ; 126(5): 376-80$

31. El Dib RP, Atallah AN, Andriolo RB. Mapping the Cochrane evidence for decision making in health care. J Eval Clin Pract. 2007;13(4):689-92.

32. Ministério da Saúde (BR). Diretrizes metodológicas Elaboração de revisão sistemática e metanálise de estudos observacionais comparativos sobre fatores de risco e prognóstico. Brasília: Ministério da Saúde; 2014.

33. Stroup DF, Berlin JA, Morton SC, Olkin I, Williamson GD, Rennie D, et al. Meta-analysis of observational studies in epidemiology: a proposal for reporting. Meta-analysis Of Observational Studies in Epidemiology (MOOSE) group. JAMA. 2000;283(15):2008-12.

34. Moat KA, Lavis JN. Supporting the use of research evidence in the Americas through an online "one-stop shop": the EVIPNet VHL. Cad Saude Publica. 2014;30(12):2697-701.

35. Ministério da Saúde (BR), Secretaria de Ciência, Tecnologia e Insumos Estratégicos. Departamento de Ciência e Tecnologia. Avaliação de tecnologias em saúde: institucionalização das ações no Ministério da Saúde. Rev Saúde Pública. 2006;40(4): 743-7.

\section{Endereço do primeiro autor:}

Patrick Alexander Wachholz

Universidade Estadual Paulista - UNESP

Avenida Prof. Mário Rubens Guimarães Montenegro, s/n

Campus de Botucatu

CEP: 18618-687 - Botucatu - SP - Brasil

E-mail: drpatrick.mdmail@gmail.com

\section{Endereço para correspondência:}

Paulo Jose Fortes Villas Boas

Universidade Estadual Paulista - UNESP

Avenida Prof. Mário Rubens Guimarães Montenegro, s/n

Campus de Botucatu

CEP: 18618-687 - Botucatu - SP - Brasil

E-mail: pvboas@fmb.unesp.br 